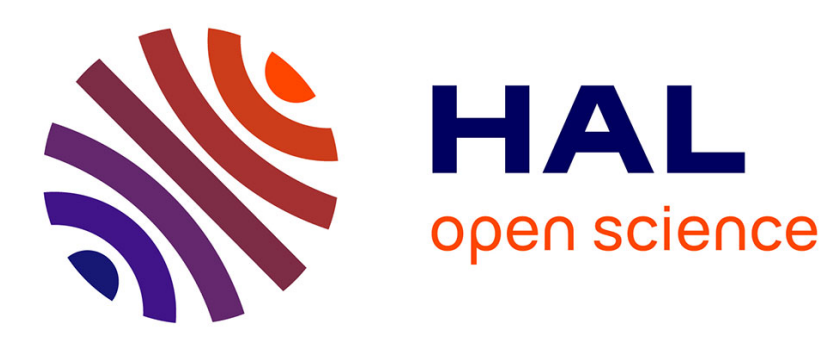

\title{
Observation of Bulk Phase Separation and Coexistence in a Sheared Micellar Solution
}

\author{
Philippe Boltenhagen, Yuntao Hu, E F Matthys, D J Pine
}

\section{To cite this version:}

Philippe Boltenhagen, Yuntao Hu, E F Matthys, D J Pine. Observation of Bulk Phase Separation and Coexistence in a Sheared Micellar Solution. Physical Review Letters, 1997. hal-02407754

\section{HAL Id: hal-02407754 \\ https://hal.science/hal-02407754}

Submitted on 12 Dec 2019

HAL is a multi-disciplinary open access archive for the deposit and dissemination of scientific research documents, whether they are published or not. The documents may come from teaching and research institutions in France or abroad, or from public or private research centers.
L'archive ouverte pluridisciplinaire HAL, est destinée au dépôt et à la diffusion de documents scientifiques de niveau recherche, publiés ou non, émanant des établissements d'enseignement et de recherche français ou étrangers, des laboratoires publics ou privés. 


\title{
Observation of Bulk Phase Separation and Coexistence in a Sheared Micellar Solution
}

\author{
Philippe Boltenhagen, ${ }^{1, *}$ Yuntao Hu, ${ }^{1}$ E. F. Matthys, ${ }^{2}$ D. J. Pine ${ }^{1,3}$ \\ ${ }^{1}$ Department of Chemical Engineering, University of California, Santa Barbara, California 93106 \\ ${ }^{2}$ Department of Mechanical Engineering, University of California, Santa Barbara, California 93106 \\ ${ }^{3}$ Department of Materials, University of California, Santa Barbara, California 93106
}

(Received 3 April 1997)

\begin{abstract}
We observe a shear-flow-induced phase transition in wormlike micelle solutions which depends on whether the applied stress or the applied shear rate is held constant. When the shear stress is held constant, the system macroscopically phase separates into two coexisting phases with a single stable interface between them. By contrast, when the shear rate is held constant, the system still undergoes a phase transition, but the steady states are homogeneous and no coexistence is observed. This leads to dramatically different rheological behavior and unusual long-lived metastable states. [S00319007(97)04155-0]
\end{abstract}

PACS numbers: $83.50 . \mathrm{By}, 83.50 . \mathrm{Qm}, 83.70 . \mathrm{Hq}$

Many complex fluids undergo structural transitions when subjected to shear flow. Some illustrative examples include liquid crystals, where shear flow can induce an isotropic-to-nematic or a nematic-to-smectic- $A$ transition [1], and surfactant systems, where shear flow can induce a lamellar-to-onion [2] or a micelle-to-vesicle transition. Shear-induced separation into polymer-rich and polymerpoor regions has also been reported in semidilute polymer solutions $[3,4]$. Because of the close analogy with equilibrium phase transitions, shear-flow-induced transitions are frequently described as nonequilibrium phase transitions.

Recently, there has been increasing interest in shearinduced phase transitions, and especially in inhomogeneous steady states which may exist between two coexisting phases. Whether or not coexistence is observed depends on the constitutive relation between stress and shear rates for the fluid and on the external constraints imposed on the fluid. For example, Cates et al. [5] suggest that when the constitutive relation is multivalued, such that several different shear rates are consistent with a single shear stress, the system may separate into two bands, with one less viscous than the other, in order to preserve the imposed average shear rate for the system. Other inhomogeneous steady states may also be possible, as suggested recently by Olmsted and Lu [6] and by Porte et al. [7]. Like Cates et al., Olmsted and Lu emphasize the importance of the imposed external constraints in determining whether or not coexistence occurs.

In this Letter, we report a shear-induced phase transition in wormlike micelle solutions in which dramatically different steady states are observed depending on whether the applied stress or the applied shear rate is controlled. In contrast to the scenario suggested by Cates et al. [5], the system macroscopically phase separates into two coexisting phases with a single stable interface between them when the imposed shear stress is held constant. When the average shear rate is held constant, the system still undergoes a phase transition, but the steady states are homogeneous and no coexistence is observed. That is, the system chooses one phase or the other, depending on the magnitude of the applied shear rate. Moreover, unlike the scenarios proposed by Cates et al. and by Olmsted and $\mathrm{Lu}$ [6], the transition we report involves shear thickening rather than shear thinning.

Our system consists of a commercial ionic surfactant, tris (2-hydroxyethyl) tallowalkyl ammonium acetate (TTAA-Ethoquad T/13-50 from AKZO Chemicals, molecular weight $454 \mathrm{~g} / \mathrm{mole}$ ), with an equimolar concentration of the salt, sodium salicylate, dissolved in water. For the work reported here, the concentration of the surfactant and salt were both $7.5 \mathrm{mM}$. This system belongs to a class of surfactant solutions which form very long wormlike micelles at very low surfactant concentrations. These micelles resemble polymers physically, but form and break spontaneously and thus constitute a kind of dynamic or "living" polymer solution. Perhaps the most remarkable property of these systems is that they become much more viscous when sheared above a critical shear rate. Moreover, the transition to the viscous state can take a considerable time to occur, from a few seconds to more than ten minutes, and is poorly understood. Our measurements imply that the increase in viscosity is the result of a dynamical nonequilibrium phase transition.

Experiments were performed in a transparent glass Couette cell attached to a commercial rheometer capable of measurements under conditions of constant shear stress or constant shear rate. The diameters of the inner and outer cylinders were 24.00 and $25.91 \mathrm{~mm}$, respectively, thus forming a gap of slightly less than $1 \mathrm{~mm}$. We obtained images of the flowing region using a visualization technique called light scattering microscopy (LSM) [8]. To obtain LSM images, the gap was illuminated by a sheet of light from a laser such that the vector normal to the sheet pointed in the flow direction. Light scattered through approximately $90^{\circ}$ from the sheet of light was magnified and collected by a CCD video camera so that an image of the flowing region, approximately $1 \mathrm{~mm} \times 2 \mathrm{~mm}$ in size, was formed. Bright regions in the LSM images originate 
from higher-than-average fluctuations in the micelle concentration.

A typical experiment consisted of the following protocol. To a well equilibrated sample, a constant shear stress or shear rate was applied starting at time $t=0$. The rheological parameters (shear rate and stress) and the LSM images were recorded continuously as a function of time until the steady state was achieved. This simultaneous measurement of rheological parameters and LSM images allowed us to directly correlate rheological changes in the system with shear-induced structural and phase transitions. All experiments were performed at $22.5 \pm 0.3{ }^{\circ} \mathrm{C}$.

First we describe our observations for the experiments performed under constant applied stress. In these experiments, the viscosity decreases as the stress increases for applied stresses of less than $0.45 \mathrm{~Pa}$. When the stress is increased above $0.45 \mathrm{~Pa}$, the measured viscosity increases and a brighter "white" shear-induced phase (SIP) begins to appear at the inner cylinder. The SIP grows and the measured viscosity increases for a period of time (typically many minutes) until a steady state is achieved in which the new SIP coexists with the preexisting darker phase. In Fig. 1, we show LSM images obtained after the solution reached steady state for three different levels of stress between 0.5 and 1.0 Pa. We also show the corresponding apparent viscosity for each level of stress as a function of time. Each light scattering image

(a)

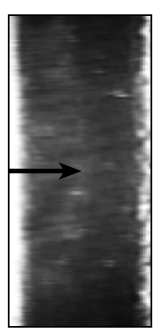

$0.5 \mathrm{~Pa}$

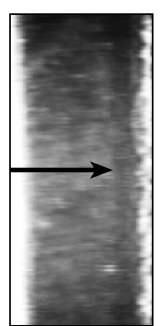

$0.7 \mathrm{~Pa}$

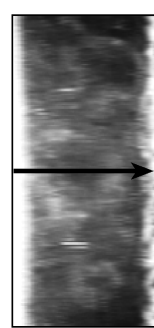

$1.0 \mathrm{~Pa}$

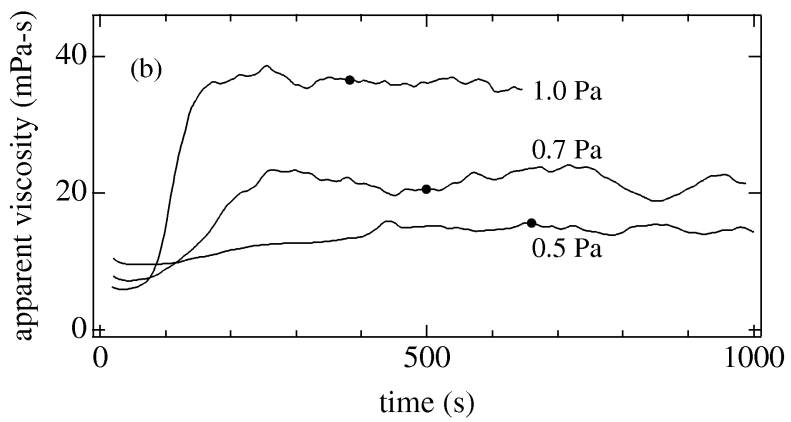

FIG. 1. (a) Scattering images obtained at various shear stresses for a $7.5 / 7.5 \mathrm{mM}$ TTAA/NaSal solution in the steady state. The SIP (bright phase) grows from the inner cylinder (left side). Arrows indicate the boundary of the shear-induced phase as characterized by the increased scattering intensity. (b) Time evolution of the apparent viscosity after the stress is applied at $t=0 \mathrm{~s}$. Circles on each curve indicate the stress and time corresponding to the images in (a). corresponds to a cross section across the gap of the Couette cell with the inner cylinder on the left. Each image shows the brighter SIP on the left side of the image with the SIP occupying an increasing fraction of the gap as the shear stress increased. This SIP is more viscous than the dark phase and appears only when the apparent viscosity begins to increase [9].

The most striking feature of the first two images in Fig. 1 is that the SIP occupies only a fraction of the gap. Moreover, the boundary between the two phases is very well defined and remains stable indefinitely, although its position fluctuates by about $10 \%$ for a given level of stress. When the stress is increased from 0.5 to $0.7 \mathrm{~Pa}$, as it is in the first two images in Fig. 1, the interface moves to the right as more SIP is created at the expense of the dark phase. Thus, we observe coexistence of two phases which are separated by a single stable interface whose position depends on the level of stress. Note that when the stress is increased to $1 \mathrm{~Pa}$, the SIP fills the entire gap, as shown in the third image. The gap remains filled with the SIP when the stress is increased beyond $1 \mathrm{~Pa}$. By contrast, no SIP was formed in the gap when the stress remained below $0.5 \mathrm{~Pa}$ even after the flow was applied for as long as 1 hour.

Figure 2 shows LSM results for measurements performed under constant applied shear rate. When the shear rate is below a critical value $\left(\sim 39 \mathrm{~s}^{-1}\right.$ in this case), no stable SIP is formed, as shown by the image obtained at $30 \mathrm{~s}^{-1}$. By contrast, when the shear rate is increased

(a)

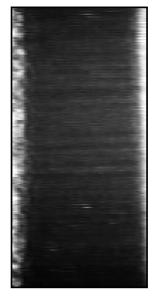

$30 s^{-1}$

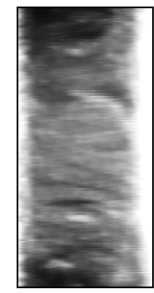

$60 s^{-1}$

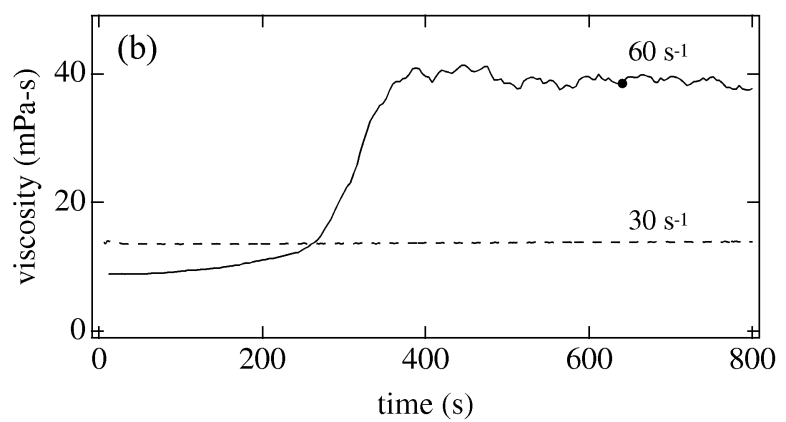

FIG. 2. (a) Scattering images obtained at shear rates below and above the critical shear rate for a 7.5/7.5 mM TTAA/NaSal solution in the steady state. The shear-induced phase is characterized by increased and fluctuating scattering intensity. (b) Time evolution of the apparent viscosity after the shear is applied at $t=0 \mathrm{~s}$. The circle on the viscosity curve indicates the position corresponding to the second image in (a). 
above the critical shear rate, the new SIP phase is formed and grows to fill the gap, as shown by the image obtained at $60 \mathrm{~s}^{-1}$. Thus, there is no steady-state coexistence between the SIP and the original dark phase. The gap is either void of the SIP or filled with the SIP. Thus, coexistence is observed under constant applied shear stress but not under constant applied shear rate.

By carrying out the step-stress and step-shear-rate measurements over a wide range of stresses and shear rates, we obtained the steady-state rheological behavior for this solution, as shown in Fig. 3, where we plot the steady-state shear stress vs apparent shear rate. The inset shows the apparent viscosity vs apparent shear rate. The data obtained at constant shear rate are similar to those reported for other micellar solutions [10-12]; the solution shear thins at low shear rates, then exhibits a discontinuous increase in viscosity (or stress) at a critical shear rate, and eventually shear thins again.

By contrast, when a constant stress is applied to the sample, the stress/shear-rate curve does not exhibit a discontinuous increase in viscosity but evolves continuously. In place of the discontinuous increase in viscosity, a reentrant region appears for stresses between approximately 0.5 and $1.5 \mathrm{~Pa}$, as shown in Fig. 3. Within the reentrant region or "loop," the shear rate first decreases with the increasing stress, reaches a minimum, and then increases again. Outside the loop, the constant stress and constant shear rate data coincide.

It is important to note that within the reentrant region, the shear rate plotted in Fig. 3 represents the mean shear rate of the inhomogeneous solution and does not correspond to the shear rates of the individual phases. In the reentrant regime, the shear rate in the viscous SIP is much smaller and the shear rate for the dark phase is

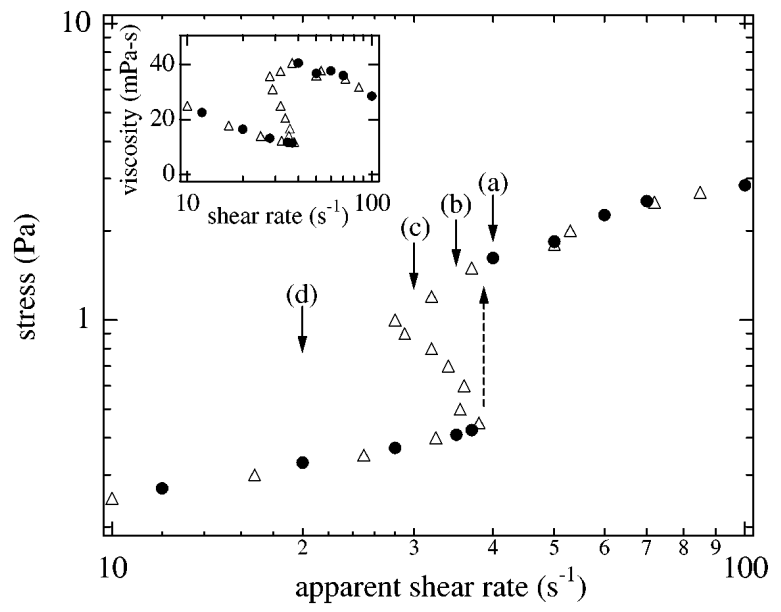

FIG. 3. Steady-state stress/shear-rate behavior obtained under constant applied shear stress (open triangles) and constant applied shear rate (solid circles) for a 7.5/7.5 mM TTAA/NaSal solution. The lettered arrows indicate the final shear rate in a series of quench experiments described in the text. The inset shows the same data plotted as apparent viscosity vs apparent shear rate. significantly greater than the mean shear rate. Thus, the constitutive relation for stress vs shear rate is multivalued when there is coexistence; that is, there are (at least) two values of the shear rate for a single value of the stress.

The origin of the difference in the stress/shear-rate behavior is evident from the LSM results. Under constant shear rate, the discontinuous increase in stress at the critical shear rate is caused by the discontinuous increase in the thickness of the SIP, which is more viscous than the dark phase; that is, the viscous SIP either fills the entire gap (above the critical shear rate) or is not present at all (below the critical shear rate), which results in a discontinuous change in the measured viscosity. By contrast, under constant shear stress, the continuous increase of stress in the reentrant region is caused by the continuous increase in the thickness of the SIP.

The filling of the gap by the SIP under constant applied shear rate is the result of a feedback mechanism that drives the system towards the viscous state. When the shear rate is increased above the critical value, the SIP begins to form at the inner cylinder [8]. The SIP is more viscous than the dark phase, and since the average shear rate is maintained at a constant value, the stress increases. This promotes the formation of more SIP which, once again, increases the stress. This process continues until the entire gap is filled with the viscous SIP.

By contrast, when the shear stress is held constant the feedback mechanism tends to stabilize the system. When the viscous SIP begins to form, the shear rate decreases, since the applied stress is held constant. The decreasing shear rate has a stabilizing effect on the system so that a coexisting state is possible.

In the reentrant region, i.e., for shear rates between approximately 28 and $39 \mathrm{~s}^{-1}$, the stress/shear-rate curve obtained under controlled stress conditions exhibits three values of stress which are consistent with a single shear rate. Which of these three stresses represents the stable steady-state value? For increasing shear rate, it is always the branch that corresponds to the smallest stress. But what happens if the system is prepared under constant shear rate conditions above the critical shear rate, and is then quenched to a shear rate in the reentrant region? We addressed this question with a series of quench experiments.

Figure 4 shows the temporal evolution of stress after the shear rate is suddenly decreased from $60 \mathrm{~s}^{-1}$ to various lower shear rates. When the shear rate is quenched to $40 \mathrm{~s}^{-1}$, which is just above the critical value, the stress remains at a high level and does not show any further decrease after a relatively short relaxation time of about one minute as shown in curve $(a)$. When the shear rate is decreased from 60 to $35 \mathrm{~s}^{-1}$, which is slightly below the critical shear rate and within the reentrant regime, the stress remains at a high level, consistent with the highest branch of the stress/shear-rate curve, for about $500 \mathrm{~s}$ and then slowly decreases to a lower level, as shown in curve $(b)$. The ultimate value of shear stress 


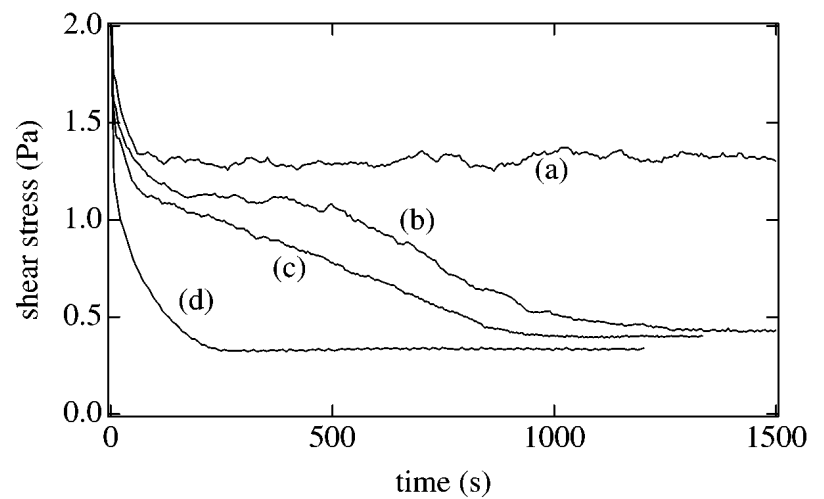

FIG. 4. Time evolution of stresses after the shear rate is decreased from $60 \mathrm{~s}^{-1}$ to various lower shear rates in a 7.5/7.5 mM TTAA/NaSal solution. Before the quench, a shear rate of $60 \mathrm{~s}^{-1}$ was applied for $600 \mathrm{~s}$ so that the steady state is reached. The letters refer to the different quenching levels indicated in Fig 3.

is consistent with the lowest branch of the stress/shearrate curve in Fig. 3. When the shear rate is decreased from 60 to $30 \mathrm{~s}^{-1}$, which is still within the reentrant regime, the stress decreases somewhat more rapidly but still very slowly as shown in curve $(c)$. Once again, the stress ultimately decays to the lowest branch of the stress/shear-rate curve. Finally, when the shear rate is further decreased to $20 \mathrm{~s}^{-1}$, which is below the reentrant regime, the stress decreases much more rapidly to the low level. These quench experiments indicate that while there are some long-lived metastable states, the system appears to choose the lowest stress, least dissipative state available to it if given sufficient time (20 $\mathrm{min}$ in some cases).

LSM images give additional insight into the decay of the shear-induced phase. When the shear rate is quenched from 60 to $30 \mathrm{~s}^{-1}$ [curve (c) in Fig. 4], the SIP does not retreat initially, but continues to fill the entire gap, in spite of the fact that the stress decreases with time. After a while, voids begin to appear in the SIP. Some time later, the SIP retreats, starting from the outer cylinder and ultimately reaching the inner cylinder. Thus, two process appear to occur: (1) a gradual dissolution in the bulk SIP, and (2) the retreat of the SIP boundary at late times, which is the reverse of the formation process.

Shear thickening, with discontinuities in the rheological data like that displayed in Fig. 1, has been observed in other solutions under constant shear rate [8,10-12]. Reentrant behavior has also been observed using devices which approximate constant stress conditions [13]. However, the different behavior of these systems under constant stress vs constant shear rate conditions was not appreciated. Nevertheless, these other experiments suggest that the phenomena we observe using the TTAA/NaSal system are common to a wide variety of surfactant systems at low concentrations. Indeed, we have made a few measurements on the hexadecyltrimethylammonium-bromide and NaSal system and find similar behavior. Thus, we believe these results can provide insight into shear thickening in a large number of low-concentration wormlike micellar solutions.

While there remain many questions about the shearinduced transition in these systems, perhaps the most puzzling is the nature and structure of the shear-induced phase. At equilibrium, the system consists of wormlike cylindrical micelles. Clearly, shear flow induces changes that result in a much more viscous shear-induced phase. Preliminary experiments in our lab suggest that the SIP may be a gel. Preliminary LSM measurements indicate that there is very little if any flow within the SIP. All the flow appears to be within the dark phase or, in those cases where the SIP fills the gap of the Couette cell, within a very narrow region next to the walls of the cell. Thus, the LSM technique provides spatial information not only about the existence and location of the two phases in the shear cell, but also on the velocity distribution. The most pressing challenge, however, is to determine the microscopic structure of the shear-induced phase, how this structure leads to a much more rigid phase, and how such a structure is produced by shear flow.

The work was funded by the MRL Program of the National Science Foundation (Award No. DMR96-32716).

*Permanent address: Laboratoire d'Ultrasons et de Dynamique des Fluides Complexes, URA au CNRS No. 851, Université Louis Pasteur, 4 rue Blaise Pascal, 67070 Strasbourg, France.

[1] C. R. Safinya, E. B. Sirota, and R. J. Plano, Phys. Rev. Lett. 66, 1986 (1996).

[2] O. Diat, D. Roux, and F. Nallet, J. Phys. II (France) 3, 1427 (1993).

[3] H. Yanase et al., Rheol. Acta 30, 89 (1991).

[4] K. Migler, C. H. Liu, and D. J. Pine, Macromolecules 29, 1422 (1996).

[5] N. A. Spenley, M.E. Cates, and T.C. B. McLeish, Phys. Rev. Lett. 71, 939 (1993); M. E. Cates, T.C. B. McLeish, and G. Marrucci, Europhys. Lett. 21, 451 (1993); N. A. Spenley, X. F. Yuan, and M. E. Cates, J. Phys. II (France) 6, 551 (1996).

[6] P. Olmsted and C.-Y. D. Lu, Phys. Rev. E 56, R55 (1997).

[7] G. Porte, J.-F. Berret, and J. L. Harden, J. Phys. II (France) 7, 459 (1997).

[8] C.-H. Liu and D. J. Pine, Phys. Rev. Lett. 77, 2121 (1996).

[9] The apparent viscosity is determined using the spatially averaged or apparent shear rate, i.e., the velocity of the inner cylinder divided by the width of the gap. For homogeneous flows, the apparent shear rate and viscosity are the actual shear rate and viscosity. For inhomogeneous flows such as those shown in Fig. 1, we report the spatially averaged shear rate and viscosity.

[10] D. Ohlendorf, W. Interthal, and H. Hoffmann, Rheol. Acta 25, 468 (1986).

[11] Y. Hu, S. Q. Wang, and A. M. Jamieson, J. Rheol. 37, 531 (1993).

[12] Y. Hu and E. F. Matthys, Rheol. Acta 34, 450 (1995).

[13] L. E. Dewalt et al., in Flow-Induced Structures in Polymers (American Chemical Society, Washington, DC, 1995), pp. 263-272. 